\title{
Evaluation of Hydroxyl Radical and Alkyl-oxy Radical Scavenging Activity of Coffee by ESR Spin Trapping Method
}

\author{
Hiromi Kameya \\ National Food Research Institute, National Agriculture Research Organization, Kannondai 2-1-12, Tsukuba-shi 305-8642, Ibaraki, \\ Japan
}

\begin{abstract}
We evaluated the hydroxyl and alkyl-oxy radical scavenging activity of instant coffee using an electron spin resonance (ESR) spin-trapping method, which was based the spin-trapping reagent 5-(2,2-dimethy-1,3-propoxycyclophosphoryl)-5-methyl-1-pyrroline N-oxide (CYPMPO). In this method, very pure hydroxyl and alkyl-oxy radicals were generated by illuminating a phosphate buffer solution containing hydrogen peroxide and 2,2'-azobis (2-amidinopropane) dihydrochloride with a Hg-Xe arc lamp. The ESR adduct signal was sensitive and very stable. We concluded that instant coffee has high hydroxyl radical and alkyl-oxy radical scavenging activity.
\end{abstract}

Key words: Radical scavenging, coffee, spin trapping, electron spin resonance.

\section{Introduction}

Antioxidants in plants act as efficient radical scavengers [1]. Oxidation processes are crucial for food storage because oxidation is a major cause of chemical spoilage of food, leading to rancidity and/or deterioration of the nutritional quality, color, flavor, and texture of food [2]. Thus, the total antioxidant activity is an important factor for consideration when evaluating food quality. A highly complex antioxidant protection mechanism has evolved to protect the body's organ systems against reactive oxygen species, involving a variety of endogenous and exogenous components that eliminate free radicals. These components include nutrient-derived antioxidants, antioxidant enzymes, metal-binding proteins, and numerous other antioxidant phytonutrients that are present in a wide variety of plant foods [2].

Prior et al. [3] reviewed some of the basic methods of measuring the antioxidant activity of foods, and three methods are recommended for standardization:

Corresponding author: Hiromi Kameya, senior researcher, research field: food science. the oxygen radical absorbance capacity (ORAC), Folin-Ciocalteu, and the 6-hydroxy-2,5,7,8-tetramethylchroman-2-carboxylic acid (Trolox) equivalent antioxidant activity methods. Although several methods have been proposed for the determination of the antioxidant's free radical scavenging activity, the ORAC method is gaining popularity in agricultural and nutritional sciences.

Electron spin resonance (ESR) spectroscopy is a powerful technique for free radical evaluation [4]. Its ability to detect radicals in dry samples makes ESR spectroscopy very useful for identifying radicals in irradiated food [5] and provides a new approach for detecting the irradiation history of food [6]. This technique has been applied to measurements of radiation-induced radicals in black pepper [7] and crude Chinese medicines [8]. ESR spectroscopy can also detect radicals in liquid media. ESR spin trapping is a technique that allows the detection and identification of low concentrations of free radicals in reacting systems [9]. This method involves the trapping of a reactive free radical by an addition 
reaction to produce a more stable ESR-detectable radical, whose hyperfine coupling parameters permit identification of the initial trapped radical.

We reported an investigation of the hydroxyl [10] and alkyl-oxy [11] radical scavenging activities of plant-based food extracts using ESR. We generated highly pure hydroxyl and alkyl-oxy radicals by illuminating a phosphate buffer solution containing hydrogen peroxide $\left(\mathrm{H}_{2} \mathrm{O}_{2}\right)$ and 2,2'-azobis(2-amidinopropane) dihydrochloride (AAPH) with a $\mathrm{Hg}-\mathrm{Xe}$ arc lamp. 5-(2,2-Dimethyl-1,3-propoxy

cyclophosphoryl)-5-methyl-1-pyrroline N-oxide (CYPMPO) is a new radical trapping reagent based on a cyclic DEPMPO-type nitrone and is colorless, crystalline, and freely soluble in water. We used CYPMPO as a radical trapping reagent to effectively trap hydroxyl and alkyl-oxy radicals $[10,11]$.

Coffee contains caffeine, a pharmacologically active ingredient that has a high radical scavenging activity $[12,13]$. However, the scavenging activity of coffee extracts has not been evaluated by ESR. The radical scavenging activity of coffee extracts is affected by roast condition, temperature of water used for extraction and extraction procedure. In the present study, we evaluated the hydroxyl and alkyl-oxy radical scavenging activity of instant coffee extracts using the ESR spin trapping method.

\section{Materials and Methods}

\subsection{Reagents}

The radical reagent CYPMPO was purchased from Radical Research (Hino, Japan). All other chemicals were purchased from Wako Pure Chemical Co. (Tokyo, Japan) and were of the highest grade.

\subsection{Samples}

The instant coffee samples (A, B, C, and D) were commercially available in Japan. Per the manufacturer's recommendation, instant coffee powder $(2 \mathrm{~g})$ was dissolved in hot water $(140 \mathrm{~mL})$ to provide a coffee concentration that was assumed to be 1 unit. We prepared samples of 0.25 units to 4 units.

\subsection{Hydroxyl Radical Scavenging Activity}

A light illuminator (cat. No. RUVF-203SR, Radical Research) was employed to produce hydroxyl radicals from $\mathrm{H}_{2} \mathrm{O}_{2}$. The light source was a $200 \mathrm{~W} \mathrm{Hg}$-Xe arc lamp (Sanei Electronics, Tokyo, Japan), which delivered mainly UV light with a wavelength of 200-400 $\mathrm{nm}$. The illuminator was equipped with a quartz fiber optical guide, and its far end was mechanically fitted to a hole in front of the ESR cavity. The illuminator was equipped with a computer controllable mechanical shutter, and various illumination periods of between 0.1 and $100 \mathrm{~s}( \pm 0.01$ s) were used. The illumination conditions were optimized so that $\mathrm{H}_{2} \mathrm{O}_{2}$ was efficiently photo decomposed to generate hydroxyl radicals. Phosphate buffer solution $(80 \mu \mathrm{L}, 100 \mathrm{mM}, \mathrm{pH}$ 7.4) that contained $\mathrm{H}_{2} \mathrm{O}_{2}(50 \mu \mathrm{L}, 1 \%)$, CYPMPO $(20 \mu \mathrm{L}, 100$ $\mathrm{mM}$ ), and diethylenetriamine pentaacetic acid (DTPA) (30 $\mu \mathrm{L} 5 \mathrm{mM}$ ), was illuminated for $5 \mathrm{~s}$ using the attenuated $\mathrm{Hg}$-Xe arc lamp light. The hydroxyl radical scavenging activity was expressed as an equivalent concentration of ascorbic acid ( $\mathrm{mM} / \mathrm{mg}$ sample).

\subsection{Alkyl-oxy Radical Scavenging Activity}

A light illuminator was employed to produce free radicals from AAPH. The illumination conditions were optimized such that AAPH was efficiently photo decomposed to generate alkyl-oxy radicals. A solution of phosphate buffer $(80 \mu \mathrm{L}, 100 \mathrm{mM}, \mathrm{pH} 7.4)$ that contained AAPH $(50 \mu \mathrm{L}, 4 \mathrm{mM})$ CYMPO $(20 \mu \mathrm{L}, 100$ $\mathrm{mM}$ ) was illuminated for $5 \mathrm{~s}$ using the attenuated Hg-Xe arc lamp. The alkyl-oxy radical trapping activity was expressed as an equivalent concentration of ascorbic acid (mM/mg sample).

\subsection{ESR Measurements}

An X-band ESR spectrometer (EMX-plus, Bruker Biospin, Yokohama, Japan) equipped with $100 \mathrm{kHz}$ 
field modulation was used for ESR measurements. Typical spectrometer settings were as follows: resonance field of $\sim 3,421-3,621 \mathrm{G}$; field modulation width of $1 \mathrm{G}$; microwave power of $6 \mathrm{~mW}$; time constant of $0.1 \mathrm{~s}$. ESR spectra were accumulated at room temperature.

The typical procedure for detection of ESR radical scavenging activity was as follows. The prepared solution was loaded into a flat disposable borosilicate sample cell (Radical Research, Hino, Japan) and placed inside the ESR cavity, where it was illuminated in situ. The ESR signal was recorded immediately after stopping the illumination.

\subsection{Kinetics}

We used the method of Kohri et al. [14] to analyze our data.

The peak-to-peak intensity of the selected ESR signal of the free radical adduct was monitored in the presence or absence of the selected antioxidant (ascorbic acid). In the presence of the radical reagent (RR) and antioxidant (AOx), the following free radical (R) scavenging reactions should occur:

$$
\begin{aligned}
\mathrm{R}+\mathrm{RR} & \rightarrow \mathrm{R} \text {-adduct (rate constant: } k_{\mathrm{RR}} \text { ) } \\
\mathrm{R}+\mathrm{AOx} & \rightarrow \text { product (rate constant: } k_{\mathrm{AOx}} \text { ) }
\end{aligned}
$$

If $\mathrm{I}_{0}$ and $\mathrm{I}$ are, respectively, the ESR peak heights that occur in the presence of $\mathrm{RR}$ alone and $\mathrm{RR}+\mathrm{AOx}$, then the amount of the product in Eq. (2) is given by $\left(\mathrm{I}_{0}-\mathrm{I}\right)$. Thus, $\mathrm{I}$ and $\left(\mathrm{I}_{0}-\mathrm{I}\right)$ are proportional to $k_{\mathrm{RR}}$ $[\mathrm{R}][\mathrm{RR}]$ and $k_{\mathrm{AOx}}[\mathrm{R}][\mathrm{AOx}]$, respectively, giving

$$
\left(\mathrm{I}_{0}-\mathrm{I}\right) \mathrm{I}=k_{\mathrm{AOx}} /[\mathrm{R}][\mathrm{AOx}] / k_{\mathrm{RR}}[\mathrm{R}][\mathrm{RR}]
$$

When nearly negligible amounts of RR and AOx are consumed in this reaction, we can safely replace $[R R]$ and $[A O x]$ with $[R R]_{0}$ and $[A O x]_{0}$, respectively, where the subscript " 0 " denotes the initial concentration of the component. Eq. (3) can then be rewritten as

$$
\mathrm{I}_{0} /(\mathrm{I}-1)=\left(k_{\mathrm{AOx}} / k_{\mathrm{RR}}\right) \cdot\left([\mathrm{AOx}]_{0} /[\mathrm{RR}]_{0}\right)
$$

In conclusion, plotting $\mathrm{I}_{0} /(\mathrm{I}-1)$ against $\left([\mathrm{AOx}]_{0} /\right.$ $\left.[R R]_{0}\right)$ gives a zero-crossing line with a slope of $\left(k_{\mathrm{AOx}}\right.$ $/ k_{\mathrm{RR}}$ ). When the same radical scavenging and free radical-generating conditions are used, $k_{\mathrm{RR}}$ becomes common for all antioxidants. If $\left(k_{\mathrm{AOX}} / k_{\mathrm{RR}}\right)$ for each sample is normalized using that of the ascorbic acid standard, the result can be expressed in terms of ascorbic acid concentration.

\section{Results and Discussions}

\subsection{Measurement of Scavenging Activity}

The ESR spectrum shown in Fig. 1 demonstrates the formation of adducts between hydroxyl and alkyl-oxy radicals and CYPMPO. An aqueous solution of CYPMPO was illuminated with a $\mathrm{Hg}$-Xe arc lamp light for $5 \mathrm{~s}$ to obtain ESR signals for the stable hydroxyl radical and alkyl-oxy radical adducts of CYPMPO. The peak-to-peak intensity of the signal at $\mathrm{g}=2.0024$ was used as the value of $\mathrm{I}_{0}$ or $\mathrm{I}$ to calculate the relative radical scavenging rate $\left(k_{\mathrm{AOx}} / k_{\mathrm{RR}}\right)$. We used solutions of ascorbic acid at concentrations of $0-0.5 \mathrm{mM}$ to calculate the relative hydroxyl radical scavenging rate, as shown in Fig. 2a. We used ascorbic acid solutions at a concentration of 0-0.06 $\mathrm{mM}$ to calculate the relative alkyl-oxy radical scavenging rate, as shown in Fig. 2 b.

\subsection{Instant Coffee Manufacturing Method and Scavenging Activity}

Sample A contained instant coffee that was manufactured using a spray-drying method, while B, $\mathrm{C}$, and $\mathrm{D}$ were manufactured using a freeze-drying method. Sample A was the lowest priced, and the cost increased for each of $\mathrm{B}, \mathrm{C}$, and D. The radical scavenging activity of 1 unit of each sample is shown in Fig. 3. The radical scavenging activity of samples manufactured by freeze-drying increased with cost. Moreover, Sample A, which was manufactured by spray-drying, showed a higher radical scavenging activity than B and C. In general, the price of instant coffee manufactured by freeze-drying is higher than that made by spray-drying. Therefore, we suggest that the radical scavenging activity of instant coffee depends 

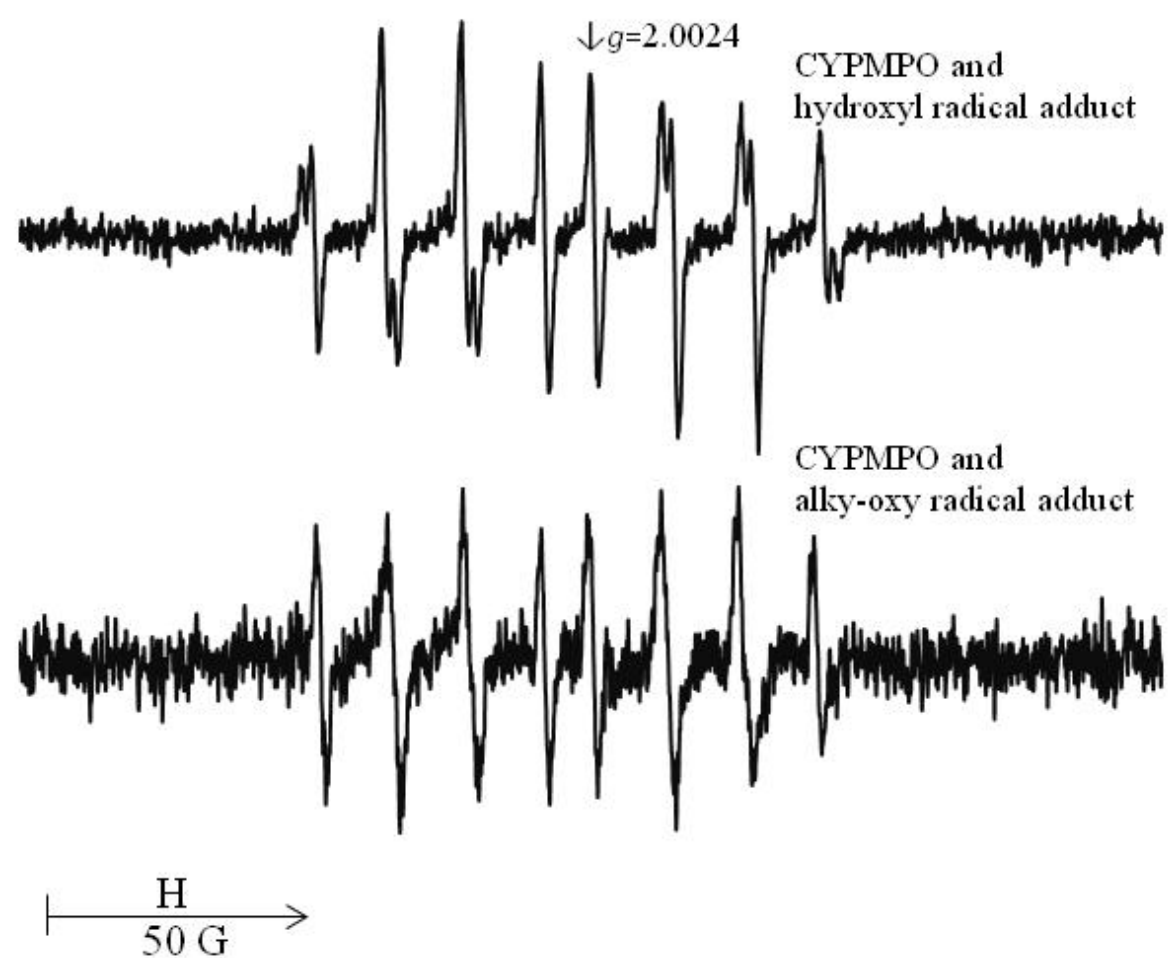

Fig. 1 ESR spectrum of the radical adducts of hydroxyl and alkyl-oxy radicals with CYPMPO. The peak-to-peak intensity of the line at $\mathrm{g}=\mathbf{2 . 0 0 2 4}$ was adopted as $\mathrm{I}_{0}$ or $\mathrm{I}$ for calculations of the relative radical scavenging rate.

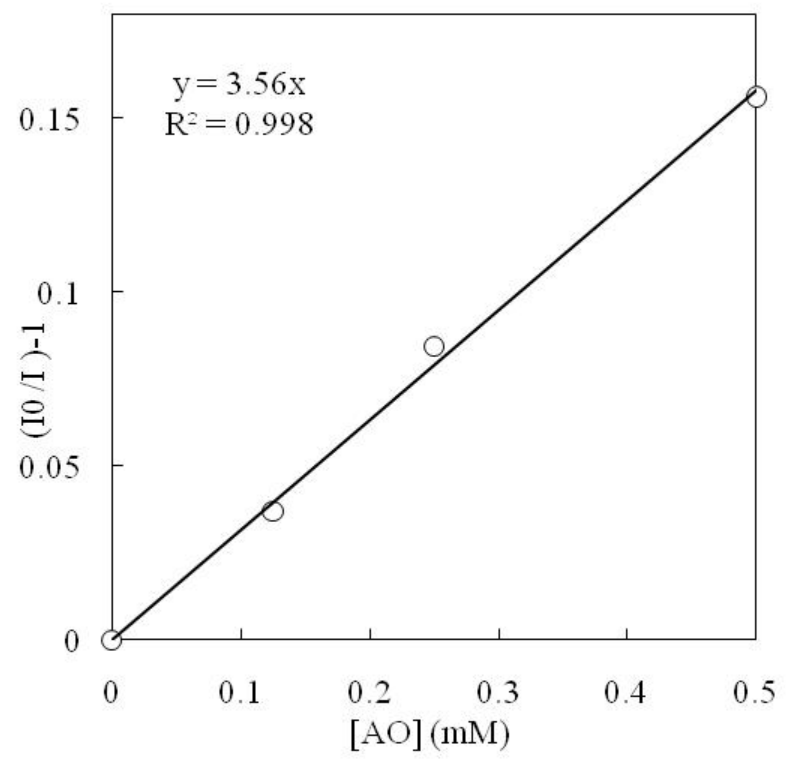

(a) hydroxyl radical

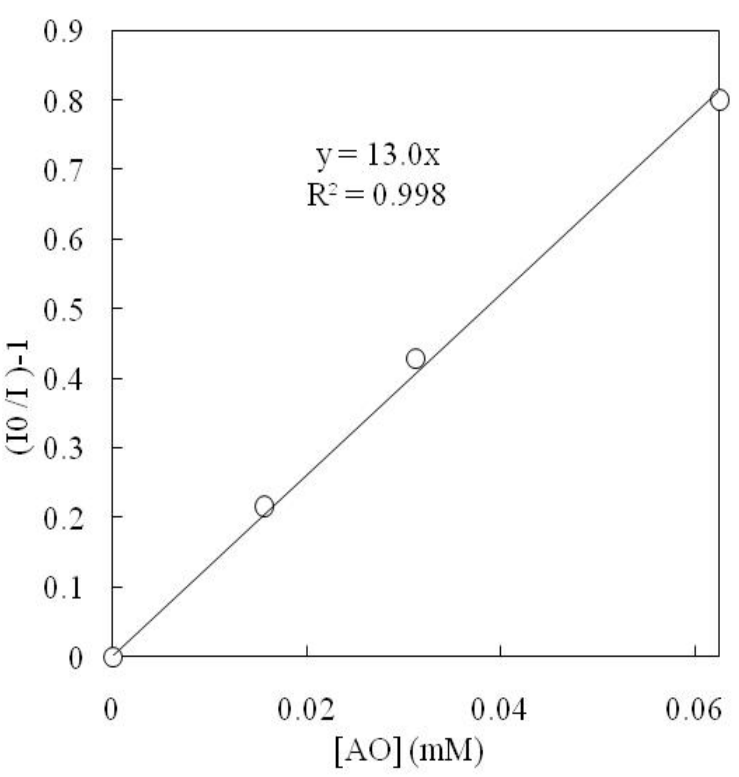

(b) alkyl-oxy radical

Fig. 2 A plot of $\left(\mathrm{I}_{0} / \mathrm{I}\right)-1$ versus [AO $]_{\mathrm{x}}$, where $\mathrm{I}_{0}$ and $\mathrm{I}$ denote the ESR signal intensity in the absence and presence of ascorbic acid. [AO] is the ascorbic acid concentration (mM). 

Activity of Coffee by ESR Spin Trapping Method

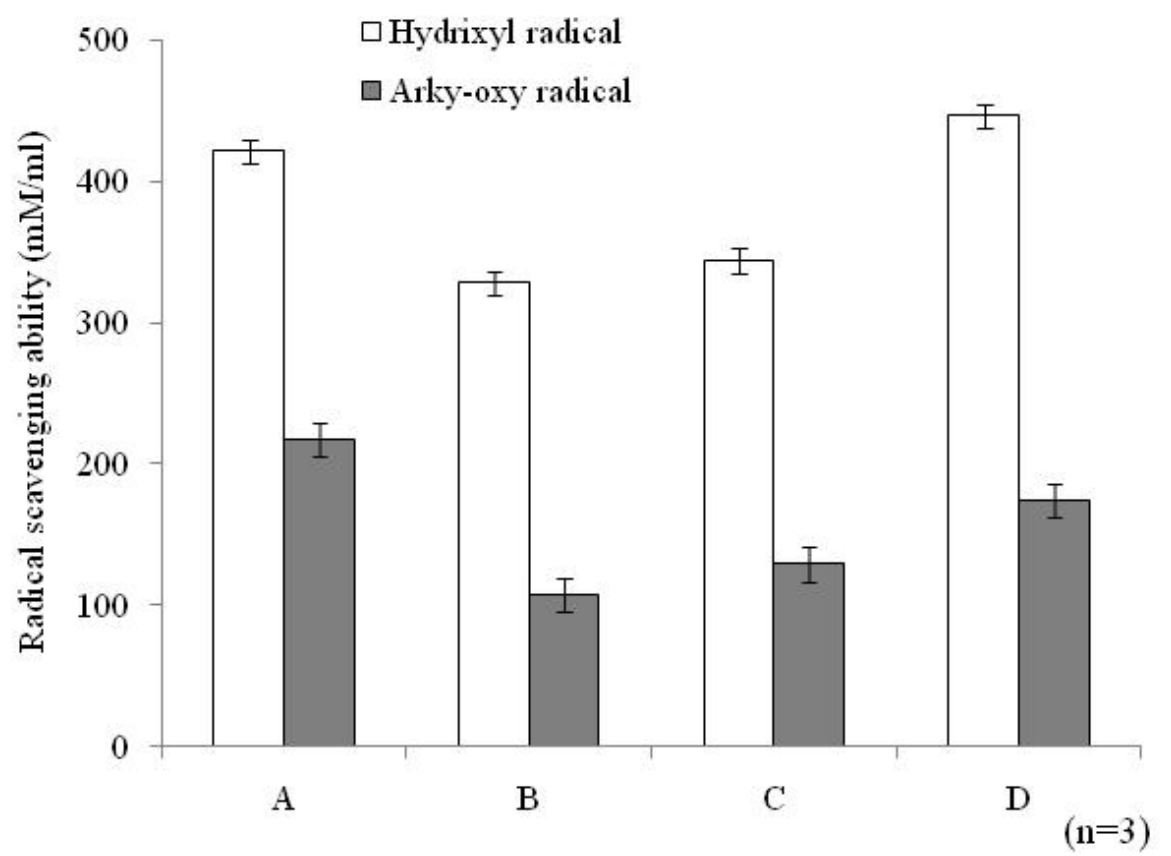

Fig. 3 The hydroxyl and alkyl-oxy radical scavenging activity of instant coffee. The activity is shown as ascorbic acid equivalent concentration $(\mathrm{mM} / \mathrm{mL})$. Three sample measurements were taken.
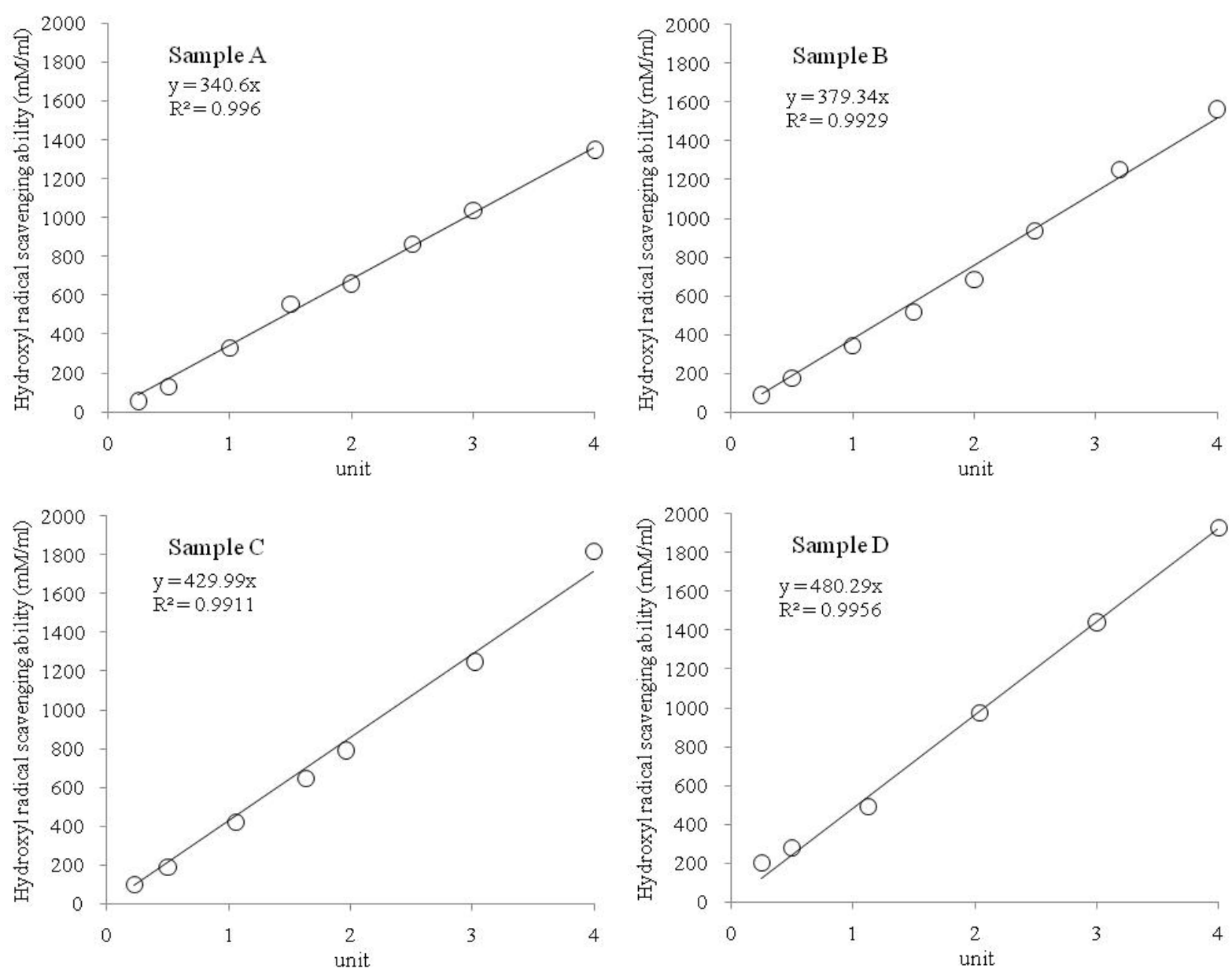

Fig. 4 Plot of hydroxyl radical scavenging activity $(\mathrm{mM} / \mathrm{mL})$ and instant coffee concentration (unit) of sample $A, B, C$, and D. 

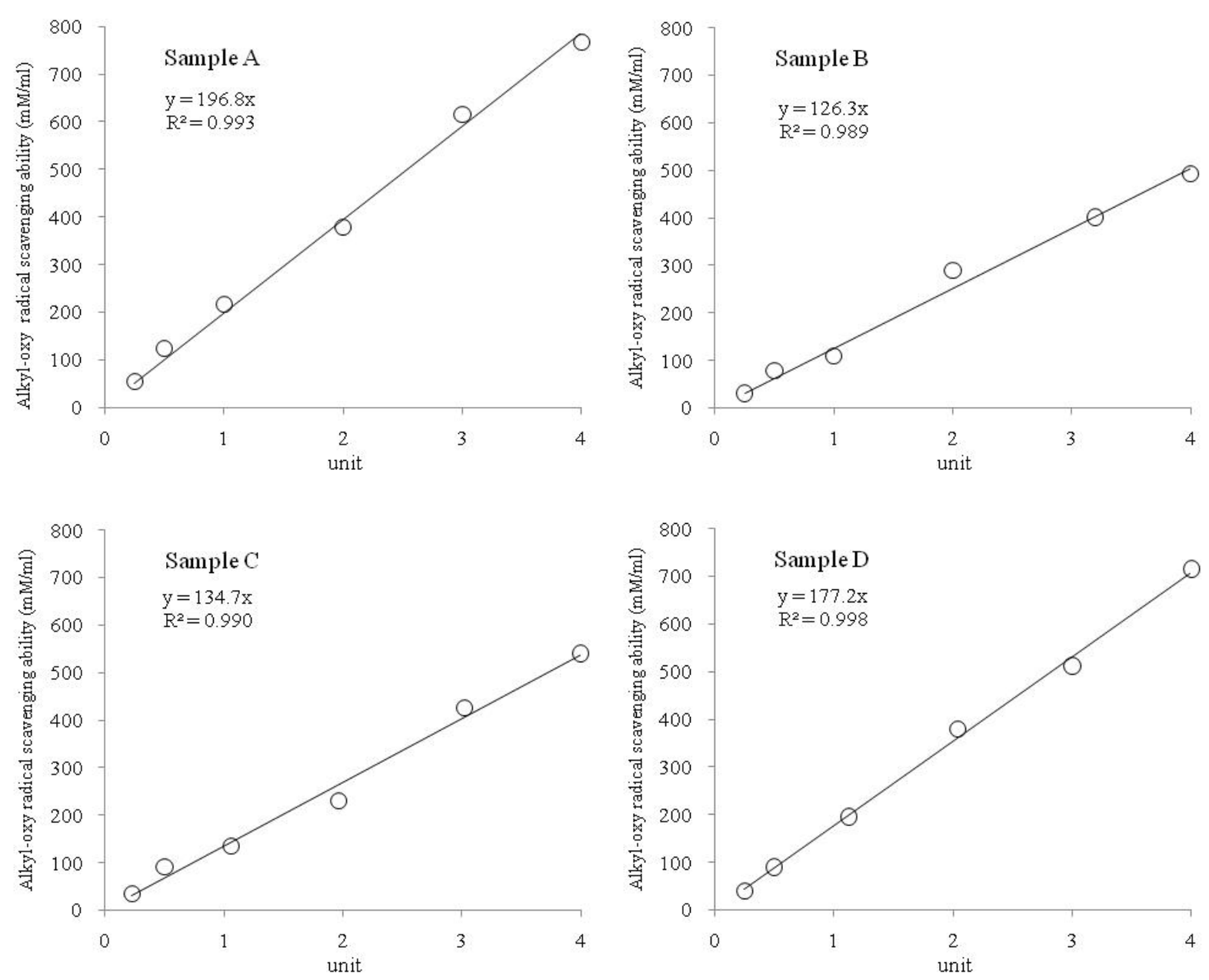

Fig. 5 Plot of alkyl-oxy radical scavenging activity $(\mathrm{mM} / \mathrm{mL})$ and instant coffee concentration (unit) of sample $\mathrm{A}, \mathrm{B}, \mathrm{C}$, and D.

on the quality of the coffee bean, rather than the manufacturing method.

\subsection{Concentration of Instant Coffee and Scavenging Activity}

The radical scavenging activity proportionally increased as the concentration was increased to 4 units, as shown in Figs. 4 and 5. Thus, the radical scavenging activity increases with concentration until a saturated solution is reached.

\subsection{Radical Scavenging Activity of Instant Coffee}

We previously reported the radical scavenging activity of blueberries [10, 11]. The hydroxyl and alkyl-oxy radical scavenging activities of blueberries (in equivalent ascorbic acid concentration) were 20
$\mathrm{mM} / \mathrm{mg}$ and $7 \mathrm{mM} / \mathrm{mg}$, respectively. One unit of instant coffee has $330-450 \mathrm{mM} / \mathrm{mL}$ of hydroxyl radical scavenging capacity and $100-220 \mathrm{mM} / \mathrm{mL}$ of alkyl-oxy radical scavenging capacity, in equivalent ascorbic acid concentrations. Thus, instant coffee has a radical scavenging activity of $\sim 20$ times that of blueberries. We concluded that instant coffee had very high hydroxyl and alkyl-oxy radical scavenging activity.

\section{Conclusions}

The hydroxyl and alkyl-oxy radical scavenging activities of instant coffee were examined using an ESR spin-trapping method. The ESR signal of the CYPMPO adduct was sensitive and very stable. We concluded that instant coffee has a very high 
Activity of Coffee by ESR Spin Trapping Method

scavenging activity due to the caffeine present in coffee beans.

\section{References}

[1] Moreno-Sánches, C. 2002. "Methods Used to Evaluate the Radical Scavenging Activity in Foods and Biological Systems.” Food Sci. Tech. Int. 8: 121-37.

[2] Percival, M. 1996. "Antioxidants." Clin. Nutr. Ins. 1: 1-6.

[3] Prior, R. L., Wu, X., and Schaich, K. 2005. "Standardized Methods for the Determination of Antioxidant Capacity and Phenolics in Foods and Dietary Supplements." $J$. Agric. Food Chem. 53 (8): 3101-13.

[4] Polovka, M., Brezová, V., and Šimko, P. 2007. "A Tool to Characterise the Gamma Irradiated Foods." J. Food Nutr. Res. 46: 75-83.

[5] Delincee, H. 2002. "Improvement of the ESR Detection of Irradiated Food Containing Cellulose Employing a Simple Extraction Method.” Radiat. Phys. Chem. 63: 455-8.

[6] Yordanov, N. D., and Aleksieva, K. 2004. "X- and Q-band EPR Studies on Fine Powders of Irradiated Plants. New Approach for Detection of Their Radiation History by Using Q-band EPR Spectrometry." Radiat. Phys. Chem. 69: 59-64.

[7] Ukai, M., and Shimoyama, Y. 2003. "An Electron Spin Resonance Study of Evolution of Organic Free Radicals in Irradiated Pepper during Heat Treatment.” J. Food Sci.
68: 2225-9.

[8] Nakamura, H., Ukai, M., and Shimoyama, Y. 2006. "An Electron Spin Resonance Study of Ginseng Irradiated by Gamma Ray." Spectrochimica Acta 63: 883-7.

[9] Janzen, E. G. 1984. "Spin Trapping." Methods in Enzymology 105: 188-98.

[10] Kameya, H., and Ukai, M. 2017. "New ESR Detection Method of Hydroxyl Radical Scavenging Activity of Blue Berry." J. Mat. Sci. Eng. in press.

[11] Ukai, M., Kameya, H., Nakamura, H., and Shimoyama, Y. 2009. "Radical Scavenging Activities of Plant Food of Alkyl-Oxy Radical and Superoxide Radical." Food Sci. Technol. Res. 15 (6): 619-24.

[12] Ramalakshmi, K., Rao, L. J. M., Takano-Ishikawa Y., and Goto, M. 2009. "Bioactivities of Low-Grade Green Coffee and Spent Coffee in Different in Vitro Model Systems." Food Chem. 115 (1): 79-85.

[13] Ramalakshmi, K., Kubra, I. R., and Rao, L. J. M. 2007. "Physicochemical Characteristics of Green Coffee: Comparison of Graded and Defective Beans." J. Food Sci. 72: S333-7.

[14] Kohri, S., Fujii, H., Oowada, S., Endoh, N., Sueishi, Y., Kusakabe, M., Shimmei, M., and Kotake, Y. 2009. “An Oxygen Radical Absorbance Capacity-like Assay that Directly Quantifies the Antioxidant's Scavenging Capacity against AAPH-derived Free Radicals." Analytical Biochem. 386: 167-71. 